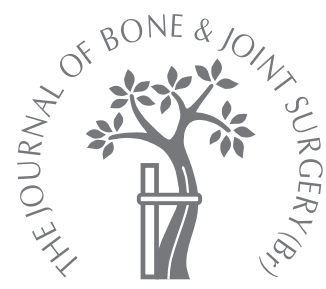

- CASE REPORT

\title{
Incomplete discoid glenoid labrum combined with a ganglion cyst of the spinoglenoid notch
}
K. J. Rhee,
K. C. Kim,
J. K. Lee,
D. S. Hwang,
H. D. Shin,
J. Y. Yang,
Y. M. Kim

\section{From Chungnam}

National University

College of Medicine,

Daejeon, Korea

\begin{abstract}
In a 41-year-old man, right-sided infraspinatus muscle weakness was associated with compression of the suprascapular nerve caused by a spinoglenoid ganglion cyst. The lesion was confirmed using electromyography and MRI. In addition, arthroscopy showed an incomplete discoid labrum. The free inner edge of the labrum was removed as in a meniscectomy of a discoid meniscus in the knee joint. Arthroscopic decompression of the cyst was performed through a juxtaglenoid capsulotomy which was left open. Neurological function recovered completely.
\end{abstract}

The glenoid labrum is variable in size and thickness, sometimes resembling a meniscus of the knee with a free inner edge projecting into the joint, and sometimes being virtually absent. ${ }^{1}$ It is recognised that abnormally large menisci in the knee, such as in a discoid lateral meniscus may occur, but only one report has described a similar abnormal morphology of the glenoid labrum. ${ }^{1}$ This report presents a rare anatomical variation resembling an incomplete discoid-shaped glenoid labrum combined with suprascapular nerve entrapment by a ganglion cyst in the spinoglenoid notch.

\section{Case report}

A 41-year-old, right-hand dominant man presented with a history of non-specific increasing shoulder pain accompanied by weakness that spread to the upper arm. Pain was provoked by overhead activities and sports, and it interrupted sleep. Physical examination revealed wasting of the infraspinatus muscle and tenderness of the shoulder posteriorly. The patient had a normal range of movement. However, weakness on resisted external rotation was observed and was graded as $3+$ out of 5 (MRC, Medical Research Council scale).

Plain radiographs revealed no abnormalities. MRI revealed a $2 \mathrm{~cm} \times 1.5 \mathrm{~cm} \times 1.5 \mathrm{~cm}$ cystic lesion in the spinoglenoid notch that had displaced the suprascapular vessels and nerve posteromedially without communicating with the glenohumeral joint. There was an unusual elongation of the glenoid labrum (Fig. 1). However, no lesion of the superior glenoid labrum extending anteriorly and posteriorly
(SLAP lesion) was evident on MRI. Electromyography (EMG) and nerve conduction studies showed an isolated lesion of the suprascapular nerve supplying the infraspinatus muscle. In addition, abnormal spontaneous activity and a reduced interference pattern were observed in the EMG of the infraspinatus muscle.

At operation the patient was positioned in the semi-reclined position. On arthroscopic examination of the glenohumeral joint, the articular surface of the glenoid was barely visible. The labrum almost covered the glenoid surface, with the exception of a $1 \mathrm{~cm}$ diameter circular area (Figs $2 \mathrm{a}$ and $2 \mathrm{~b}$ ). The projecting free inner margin of the labrum was removed and debrided using a basket and shaver, as in a meniscectomy of a discoid meniscus in the knee joint (Fig. 2c). There was no SLAP lesion, area of labral detachment, or communication with the spinoglenoid cyst on probing. A $1 \mathrm{~cm}$ capsulotomy was performed near the junction of the capsule and labrum posterosuperiorly. Dissection with a blunt probe along the glenoid neck toward the spinoglenoid notch, combined with digital pressure over the infraspinatus fossa, decompressed the cyst into the joint through the capsulotomy, which was left open (Fig. 2d).

Two years after surgery the patient has no symptoms related to the suprascapular nerve. He returned to work without restriction, and has no subjective or objective signs of weakness or wasting.

\section{Discussion}

The term incomplete discoid glenoid labrum was coined in 1997 by Lee and Harryman, ${ }^{1}$ to 


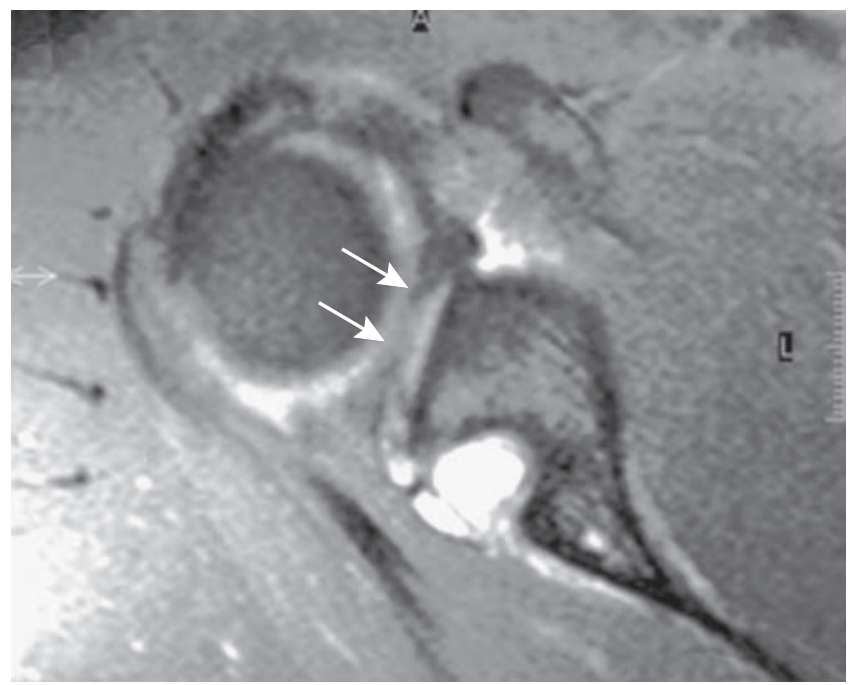

Fig. 1a

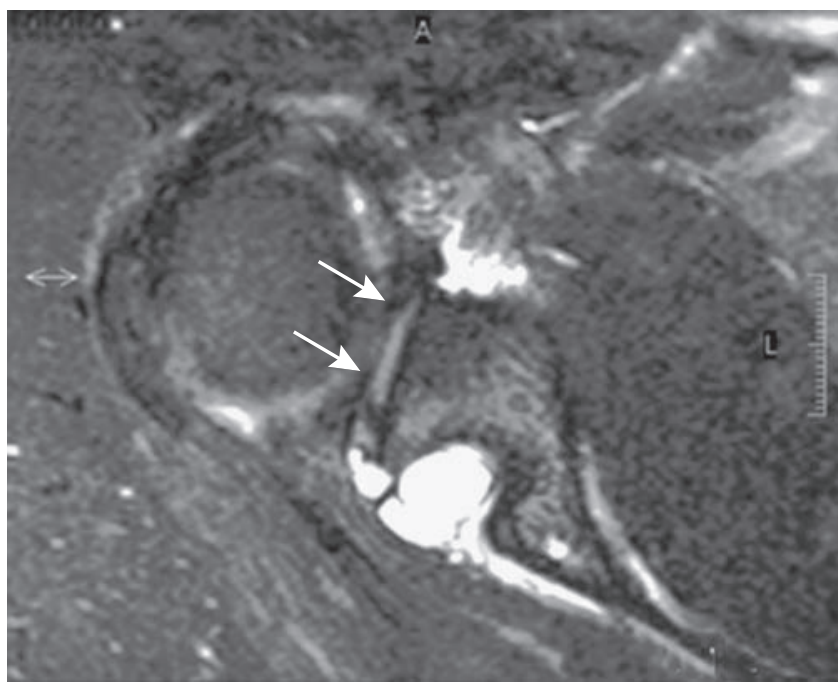

Fig. 1b

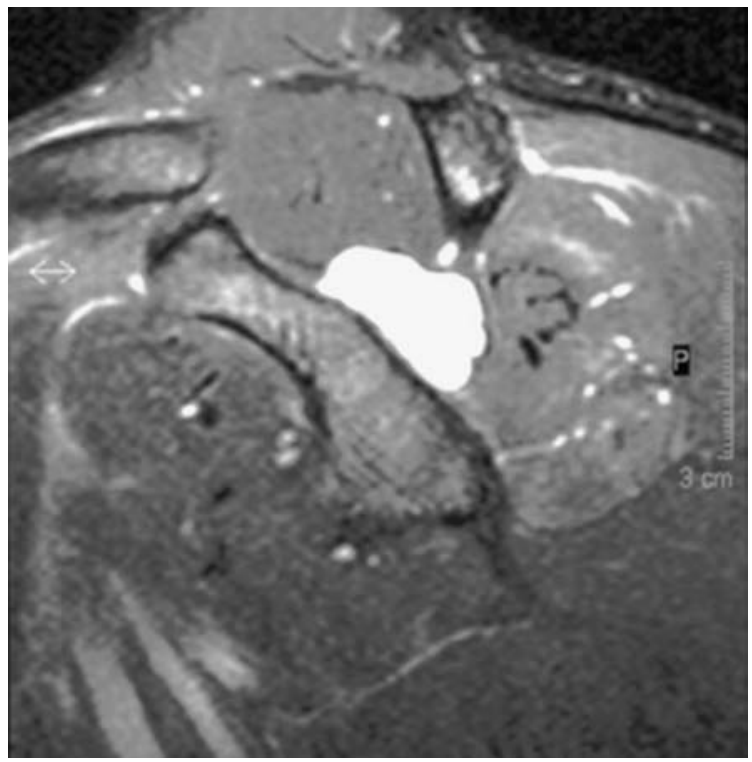

Fig. 1c

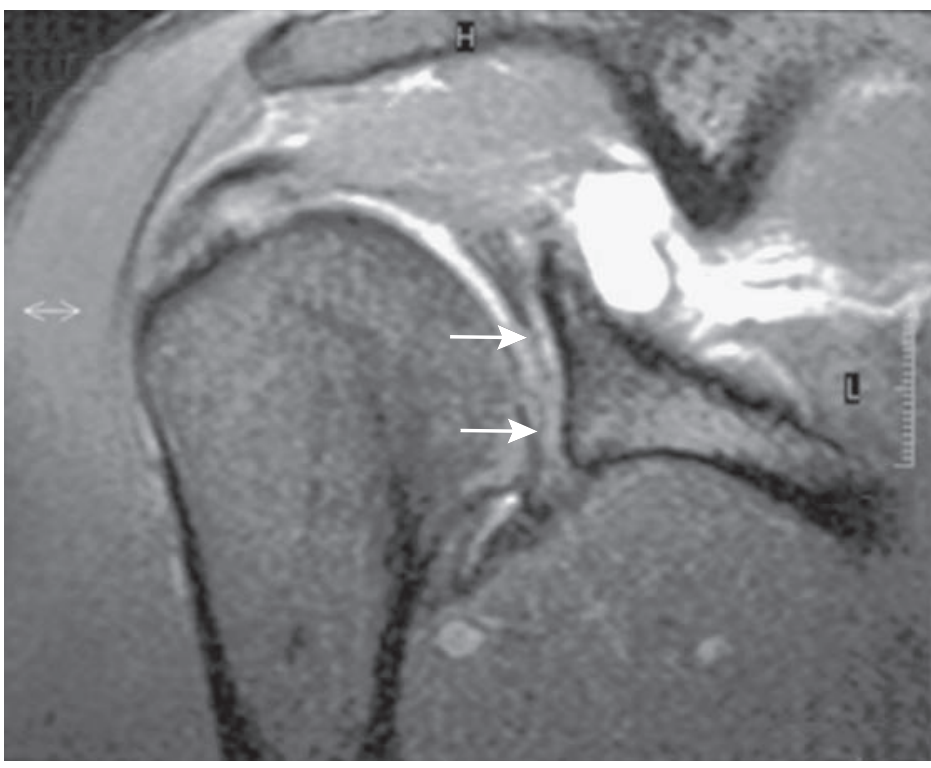

Fig. 1d

Pre-operative MR scans of the right shoulder showing a cyst at the spinoglenoid notch with no communication with the glenohumeral joint, and unusual elongation of the glenoid labrum in the glenohumeral joint (arrows indicate the elongated labrum).

describe a rare anatomical variation. The authors reported one case in a wheelchair-bound patient of superior detachment of a glenoid labrum variant resembling an incomplete discoid meniscus. Only a small central area of the articular glenoid was left exposed by the uniformly wide and thick meniscal-shaped labrum, as in our patient. However, in their patient, superior detachment of the discoid labrum and biceps origin (type II SLAP lesion ${ }^{2}$ ) required arthroscopic reattachment of the biceps and superior labrum using a staple without removing the free inner edge of the labrum.

Several authors have noted a relationship between cysts in the spinoglenoid notch and superior labral pathology, even when no labral disruption is evident on MRI. ${ }^{3-6}$
Moore et $\mathrm{al}^{4}$ observed SLAP lesions intra-operatively in 11 of 12 patients with supraglenoid cysts and suprascapular nerve entrapment, but no pre-operative MRI evidence of labral pathology. Although no superior labral pathology was observed in our patient, a rare anatomical variation resembling an incomplete discoid-shaped labrum was observed. Ianotti and Ramsey ${ }^{7}$ described three patients whose cysts were decompressed through a labral tear or by capsulotomy near the junction of the capsule and labrum if no tear was present. The ganglion was drained into the joint by manual pressure over the cyst. All three patients became pain free and recovered full strength on external rotation. In our case, a juxtaglenoid capsulotomy of 


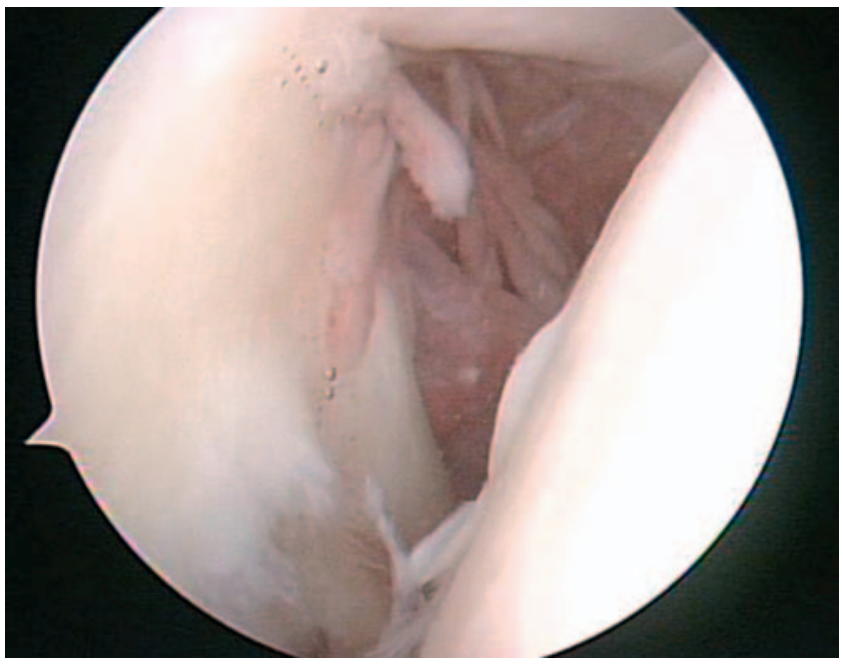

Fig. 2a

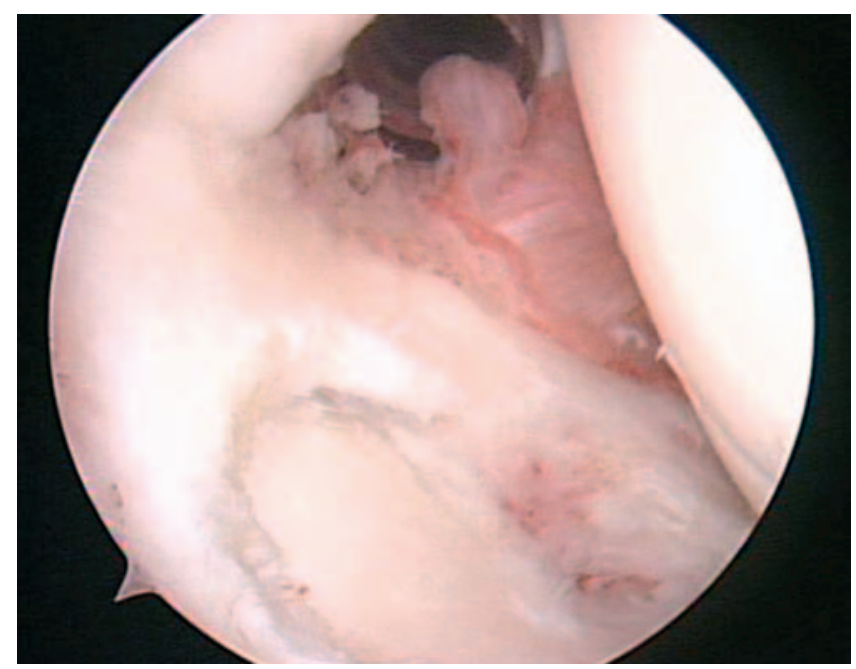

Fig. 2c

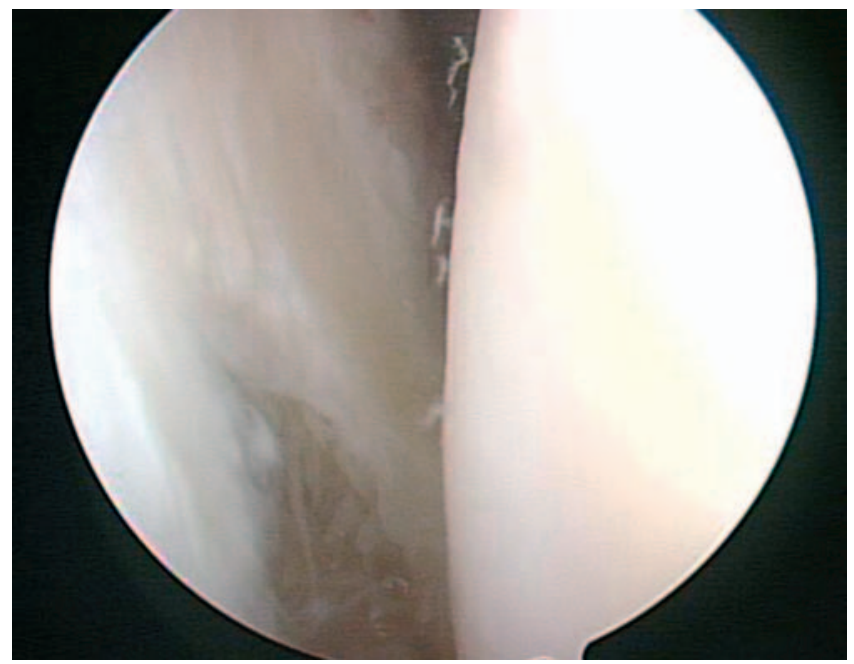

Fig. $2 b$

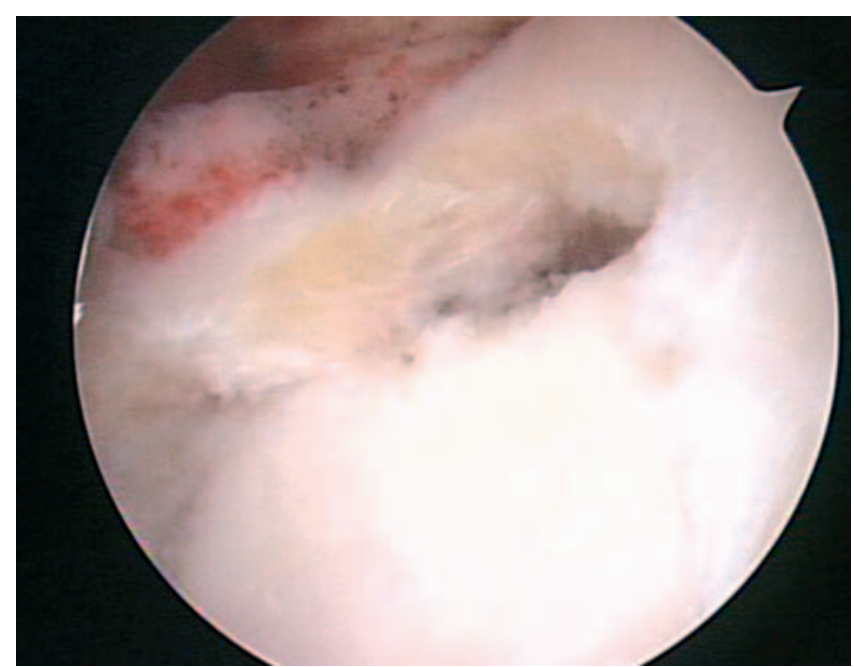

Fig. 2d

Figure $2 \mathrm{a}$ - Arthroscopic view through the posterior portal showing a labrum nearly covering the glenoid surface. Figure $2 \mathrm{~b}-\mathrm{Arth}$ roscopic view through the posterior portal showing only a small central area of articular glenoid exposed by the uniformly wide and thick meniscal-shaped glenoid labrum. Figure $2 c$ - The projecting free inner edge of the glenoid labrum was removed and debrided. Figure $2 d-$ Arthroscopic view through the anterior portal showing the decompressed cyst through a capsulotomy near the junction of the capsule and the labrum at the posterior superior glenoid, and leaving the capsulotomy open.

approximately $1 \mathrm{~cm}$ was performed to decompress the cyst, and the capsulotomy was left open.

In conclusion, we report an incomplete discoid glenoid labrum combined with a ganglion cyst at the spinoglenoid notch, which has not been reported previously. An incomplete discoid glenoid labrum may be be a further form of labral pathology associated with this condition.

No benefits in any form have been received or will be received from a commercial party related directly or indirectly to to the subject of this article.

\section{References}

1. Lee SB, Harryman DT 2nd. Superior detachment of a glenoid labrum variant resembling an incomplete discoid meniscus in a wheelchair ambulator. Arthroscopy 1997:13:511-14.
2. Snyder SJ, Karzel RP, Del Pizzo W, Ferkel RD, Friedman MJ. SLAP lesions of the shoulder. Arthroscopy 1990;6:274-9.

3. Chen AL, Ong BC, Rose DJ. Arthroscopic management of spinoglenoid cysts associated with SLAP lesions and suprascapular neuropathy. Arthroscopy 2003;19: 5-21.

4. Moore TP, Fritts HM, Quick DC, Buss DD. Suprascapular nerve entrapment caused by supraglenoid cyst compression. J Shoulder Elbow Surg 1997;6: 455-62.

5. Tirman PFJ, Feller JF, Janzen DL, et al. Association of glenoid labral cysts with labral tears and glenohumeral instability: radiologic findings and clinical significance. Radiology 1994;190:653-8

6. Ferrick MR, Marzo JM. Ganglion cyst of the shoulder associated with a glenoid labral tear and symtomatic glenhumeral instability: a case report. Am J Sports Med 1997;25:717-19.

7. lanotti JP, Ramsey ML. Arthroscopy decompression of a ganglion cyst causing suprascapular nerve compression. Arthroscopy 1996:12:739-45. 